This is a self-archived version of an original article. This version may differ from the original in pagination and typographic details.

Author(s): Bobkova, I. V.; Bobkov, A. M.; Silaev, Mikhail

Title: Spin torques and magnetic texture dynamics driven by the supercurrent in superconductor/ferromagnet structures

Year: 2018

Version: Published version

Copyright: (c) 2018 American Physical Society

Rights: In Copyright

Rights url: http://rightsstatements.org/page//nC/1.0/?language=en

Please cite the original version:

Bobkova, I. V., Bobkov, A. M., \& Silaev, M. (2018). Spin torques and magnetic texture dynamics driven by the supercurrent in superconductor/ferromagnet structures. Physical Review B, 98(1), Article 014521. https://doi.org/10.1103/physrevb.98.014521 


\title{
Spin torques and magnetic texture dynamics driven by the supercurrent in superconductor/ferromagnet structures
}

\author{
I. V. Bobkova, ${ }^{1,2}$ A. M. Bobkov, ${ }^{1}$ and M. A. Silaev ${ }^{3}$ \\ ${ }^{1}$ Institute of Solid State Physics, Chernogolovka, Moscow reg., 142432 Russia \\ ${ }^{2}$ Moscow Institute of Physics and Technology, Dolgoprudny, 141700 Russia \\ ${ }^{3}$ Department of Physics and Nanoscience Center, University of Jyväskylä, P.O. Box 35 (YFL), FI-40014 University of Jyväskylä, Finland
}

(Received 4 April 2018; revised manuscript received 17 May 2018; published 26 July 2018)

\begin{abstract}
We introduce the general formalism to describe spin torques induced by the supercurrents injected from the adjacent superconducting electrodes into the spin-textured ferromagnets. By considering the adiabatic limit for the equal-spin superconducting correlations in the ferromagnet, we show that the supercurrent can generate both the fieldlike spin-transfer torque and the spin-orbital torque. These dissipationless spin torques are expressed through the current-induced corrections to the effective field derived from the system energy. The general formalism is applied to show that the supercurrent can either shift or move the magnetic domain walls depending on their structure and the type of spin-orbital interaction in the system. These results can be used for the prediction and interpretation of the experiments studying magnetic texture dynamics in superconductor/ferromagnet/superconductor Josephson junctions and other hybrid structures.
\end{abstract}

DOI: 10.1103/PhysRevB.98.014521

\section{INTRODUCTION}

It has been commonly recognized that reducing Joule heating effects and power consumption are among the main priorities for the development of electrically controlled magnetic memory devices [1-4]. Since the first spin-transfer torque (STT) experiments [5,6], much effort has been invested to optimize the switching currents, thermal stability, and tunnel magnetoresistance of the magnetic tunnel junctions $[3,7,8]$. Thermal effects are also of the crucial importance for the operation of the other type of STT memory-magnetic racetrack memory [9-11] based on the electrical control over the domain wall (DW) motion. The progress in improving these spin memory devises depends crucially on the competition between the thermal stability of DWs and large current densities required to overcome the pinning forces $[4,12-18]$. As an alternative route to the low-power manipulation of magnetic textures, the current-driven magnetic skyrmion dynamics has attracted large interest [19-21].

In applications that require very large currents, for example, in powerful magnets, using superconducting materials have been proven to be an effective solution to eliminate Joule heating effects. In view of the energy-saving spintronics, it is quite appealing to employ the spin torques generated by the dissipationless spin-polarized superconducting currents (supercurrents). The existence of spin-polarized supercurrents is ubiquitous to the spin-textured superconductor/ferromagnet (SC/FM) hybrid structures resulting from long-range spintriplet proximity [22-25].

Recently, there have been many works studying spinpolarized supercurrents in various SC/FM systems (for the review see Refs. [26,27]). However, the supercurrent-induced spin torques have been characterized theoretically only in several model systems: in Josephson junctions through singledomain magnets [28-32], two [33-35] and three [36] FM layers, and in ferromagnetic spin-singlet [37] and spintriplet superconductors [38]. The general understanding of the supercurrent-spin texture interaction has been lacking since there is no direct connection between the above examples and practically interesting systems — bulk nonhomogeneous FMs. That is, the possibility of moving DWs and skyrmions by injecting the supercurrent in real ferromagnets has been an open question for a long time despite of the large attention to the subject.

This challenging question is addressed in the present paper. We employ the adiabatic approximation, which is widely used for the description of kinetic processes in metallic ferromagnets with spin textures including the calculation of conductivity [19] and spin-transfer torques [39] in the inhomogeneous FMs. We bring this approach to the realm of superconducting systems to describe their transport properties governed by equalspin superconducting correlations. For that, we go beyond the commonly used quasiclassical theory of hybrids [25,40], which has been designed to treat only weak ferromagnets with an exchange splitting much less than the Fermi energy. Instead of that, we employ the recently developed approach of generalized quasiclassical theory [41], which allows for the description of proximity effect in strong ferromagnets with an exchange splitting much larger than other energy scales and comparable to the Fermi energy.

We show that the spin-polarized superconducting current can induce magnetization dynamics, described in general by the Landau-Lifshitz-Gilbert (LLG) equation

$$
\dot{\boldsymbol{M}}=-\gamma \boldsymbol{M} \times \boldsymbol{H}_{\mathrm{eff}}+\frac{\alpha}{M} \boldsymbol{M} \times \dot{\boldsymbol{M}},
$$

where $\gamma=2 \mu_{B}$ is the electron gyromagnetic ratio. The second term in the r.h.s. is the Gilbert damping. The superconducting spin current $\boldsymbol{J}$ can induce two types of spin torques which can be written as the correction to effective field $-\gamma \boldsymbol{M} \times \tilde{\boldsymbol{H}}_{\text {eff }}=$ 
$\boldsymbol{N}_{\text {st }}+\boldsymbol{N}_{\text {so }}$. The first term here is the adiabatic spin-transfer torque [15,42,43], while the second term $\boldsymbol{N}_{\text {so }}$ is the spin-orbital (SO) torque $[44,45]$. The nonadiabatic (antidamping) STT [14] is not produced by the supercurrent since it breaks the timereversal symmetry of LLG equation and can be considered as a correction to the dissipative Gilbert damping [46]. That is, the antidamping STT should be connected with the quasiparticle contribution, which is beyond the scope of our present study.

The paper is organized as follows. In Sec. II, the general equations for spin dynamics and spin torques generated by supercurrent are considered. In Sec. III, we derive expressions for the spin-transfer and spin-orbital torques using the generalized quasiclassical theory. In Sec. IV, we derive the Josephson energy in SFS junctions and use it to provide an alternative derivation of supercurrent spin torques. Section V is devoted to the DW dynamics in SFS Josephson junctions induced by the supercurrent spin torques. Our conclusions are given in Sec. VI.

\section{SPIN TORQUE GENERATED BY THE SUPERCURRENT}

We use an $s-d$ model with a localized magnetization $\boldsymbol{M}$ and that of the itinerant electrons $\boldsymbol{M}_{s}=-\mu_{B} \boldsymbol{s}$, where $\boldsymbol{s}$ is the electron spin and $\mu_{B}$ is the Bohr magneton. The dynamics of localized spins is determined by the usual LLG equation with a contribution to the effective field resulting from exchange interaction with conductivity electrons [47]:

$$
\dot{\boldsymbol{M}}=-\gamma \boldsymbol{M} \times \boldsymbol{H}_{\text {eff }}+\frac{\alpha}{M} \boldsymbol{M} \times \dot{\boldsymbol{M}}-J_{\mathrm{sd}} \boldsymbol{M} \times \boldsymbol{M}_{s} .
$$

The last term here is the source of spin torque and should be found from the kinetic equation for conductivity electrons.

The kinetic theory for the conduction electrons in metals can be formulated in terms of the matrix Green's function $\breve{G}=$ $\breve{G}\left(\boldsymbol{r}_{1}, \boldsymbol{r}_{2}, t_{1}, t_{2}\right)$, which has the following explicit structure in the Keldysh space $\breve{G}=\left(\begin{array}{cc}\breve{G}^{R} & \breve{G}^{K} \\ 0 & \breve{G}^{A}\end{array}\right)$, where $\breve{G}^{R / A / K}$ are the retarder/advanced/Keldysh components. The general quantum kinetic equation reads

$$
\begin{gathered}
i\left\{\partial_{t}, \check{G}\right\}_{t}-[\hat{H}, \check{G}]_{t, \boldsymbol{r}}=\check{I}, \\
\hat{H}(t, \boldsymbol{r})=-\frac{\hat{\boldsymbol{\Pi}}_{\boldsymbol{r}}^{2}}{2 m_{F}}+(\hat{\boldsymbol{\sigma}} \boldsymbol{h}(\boldsymbol{r}, t)) \hat{\tau}_{3}-i\left(\hat{\boldsymbol{\sigma}} \hat{B} \hat{\boldsymbol{\Pi}}_{r}\right), \\
\check{I}=(\check{\Sigma} \circ \check{G}-\check{G} \circ \check{\Sigma})\left(\boldsymbol{r}_{1}, \boldsymbol{r}_{2}, t_{1}, t_{2}\right) .
\end{gathered}
$$

Here, we define the o-product as $(\hat{A} \circ \hat{B})\left(t_{1}, t_{2}\right)=$ $\int_{-\infty}^{\infty} d t \hat{A}\left(t_{1}, t\right) \hat{B}\left(t, t_{2}\right)$. The commutator is defined as $[\hat{H}, \breve{G}]_{t}=\hat{H}\left(t_{1}, \boldsymbol{r}_{1}\right) \check{G}-\check{G} \hat{H}\left(t_{2}, \boldsymbol{r}_{2}\right), \quad \hat{\boldsymbol{\Pi}}_{\boldsymbol{r}}=\nabla-i e \hat{\tau}_{3} \boldsymbol{A}(\boldsymbol{r})$, $\hat{\sigma}_{i}$ and $\hat{\tau}_{i}$ are Pauli matrices in spin and Nambu spaces, respectively. The exchange field is determined by localized moments $\boldsymbol{h}=-J_{\mathrm{sd}} \boldsymbol{M} / 2 \mu_{B}$. The last term in Eq.( 4) is the general form of a linear in momentum spin-orbit coupling (SOC) determined by the constant tensor coefficient $\hat{B}$. The collision integral in the right-hand side (r.h.s.) of Eq. (3) is given by Eq. (5). The self-energy term $\check{\Sigma}$ includes the effects related to disorder scattering as well as the off-diagonal superconducting self-energies.
The conduction electron spin polarization $s$, charge $j$ and spin $\boldsymbol{J}^{i}$ currents are given by

$$
\begin{gathered}
\boldsymbol{s}(\boldsymbol{r}, t)=-\left.\frac{i}{8} \operatorname{Tr}_{4}\left[\hat{\boldsymbol{\sigma}} \hat{\tau}_{3} \hat{G}^{K}\right]\right|_{\boldsymbol{r}_{1,2}=\boldsymbol{r}, t_{1,2}=t}, \\
\boldsymbol{j}(\boldsymbol{r}, t)=\left.\operatorname{Tr}_{4}\left[\frac{\left(\hat{\boldsymbol{\Pi}}_{\boldsymbol{r}_{1}}-\hat{\boldsymbol{\Pi}}_{\boldsymbol{r}_{2}}\right)}{8 m_{F}} \hat{\tau}_{3} \check{G}^{K}\right]\right|_{\boldsymbol{r}_{1,2}=\boldsymbol{r}, t_{1,2}=t}, \\
\boldsymbol{J}^{k}(\boldsymbol{r}, t)=\left.\operatorname{Tr}_{4}\left[\frac{\left(\hat{\boldsymbol{\Pi}}_{\boldsymbol{r}_{1}}-\hat{\boldsymbol{\Pi}}_{\boldsymbol{r}_{2}}\right)}{16 m_{F}} \hat{\sigma}_{k} \check{G}^{K}\right]\right|_{\boldsymbol{r}_{1,2}=\boldsymbol{r}, t_{1,2}=t} .
\end{gathered}
$$

The strategy of studying magnetization dynamics consists of solving the coupled LLG (2) and kinetic equations (3)(5) together with the expression for the magnetic moment (6). However, the general problem is too complicated for the analysis. In the next section, (Sec. III), we discuss the simplification of the kinetic equation using the so-called generalized quasiclassical approximation [41] adopted to treat the nonstationary problems.

Besides that, a significant simplification can be obtained in the linear response limit when the dynamics of magnetization is slow so that the characteristic frequency is small as compared to the energy gap in the quasiparticle spectrum. In this case, we can make use of the quasistationary equation for the electron magnetization, which is obtained from Eq. (3). Multiplying it by $\hat{\sigma}$ from the left and taking the trace we obtain

$$
\begin{aligned}
-\partial_{t} \boldsymbol{s}+\nabla_{j} \boldsymbol{J}_{j}= & \frac{J_{\mathrm{sd}}}{\mu_{B}}\left(\boldsymbol{M} \times \boldsymbol{M}_{s}\right) \\
& +2 m_{F}\left(\boldsymbol{B}_{j} \times \boldsymbol{J}_{j}\right)+\frac{\operatorname{Tr}_{4}[\hat{\boldsymbol{\sigma}} \check{I}]^{K}}{8} .
\end{aligned}
$$

Here, we introduce the vector $\boldsymbol{B}_{j}=\left(B_{x j}, B_{y j}, B_{z j}\right)$, which is determined by the $j$ th coordinate component of the tensor $\hat{B}$, and $\boldsymbol{J}_{j}=\left(J_{j}^{x}, J_{j}^{y}, J_{j}^{z}\right)$ is the $j$-coordinate component of the spin current (8). Next, the driving term in the LLG equation (2) can be found neglecting the term with time derivative in Eq. (9):

$$
\frac{J_{\mathrm{sd}}}{\mu_{B}}\left(\boldsymbol{M} \times \boldsymbol{M}_{s}\right)=\nabla_{j} \boldsymbol{J}_{j}-2 m_{F}\left(\boldsymbol{B}_{j} \times \boldsymbol{J}_{j}\right)-\frac{\operatorname{Tr}_{4}[\hat{\boldsymbol{\sigma}} \check{I}]^{K}}{8} .
$$

In this work, we are interested in the quasiequilibrium spin torques generated solely by the supercurrent without the contribution of nonequilibrium quasiparticles. That means the normal component of the current and the electric field are assumed to be absent. Generally, the last term with the collision integral in Eq. (10) has contributions both from the off-diagonal order parameter and the spin-orbital scattering self-energy. The present work is based on the following simplifying assumptions allowing to put $\operatorname{Tr}_{4}[\hat{\sigma} \check{I}]^{K}=0$. First, we are interested in the spin torques occurring in the normal metal ferromagnetic interlayer where the order parameter is absent. Second, the exchange splitting between spin subbands is assumed to be large enough to suppress spin-flip transitions between them. Below, we demonstrate that in this regime the first term in the r.h.s. of Eq. (10) produces the adiabatic STT $[39,42,48]$, while the second term yields the spin-orbit torque $[44,45,49]$. In order 
to find these contributions, we calculate the spin supercurrent through the spatially-inhomogeneous ferromagnet. In the next section, it is shown that in the adiabatic limit valid for the description of strong ferromagnet, this calculation can be done analytically in the most general way using the technique developed in Ref. [41].

\section{GENERALIZED QUASICLASSICAL THEORY}

\section{A. Eilenberger equation for equal-spin correlations}

To find the results in the adiabatic approximation, it is convenient to work in the local reference frame, where the spin quantization axis is aligned with the local direction of the exchange field in the ferromagnet. Then we use the transformation $\breve{G}_{\text {loc }}=\hat{U}^{\dagger} \check{G} \hat{U}$, where $\hat{U}=\hat{U}(\boldsymbol{r}, t)$ is in general the time- and space-dependent unitary $2 \times 2$ matrix that rotates the spin quantization axis $z$ to the local frame determined by the exchange field, so that $\boldsymbol{h} \| \boldsymbol{z}$.

To implement the adiabatic approximation, we introduce the equal-spin (ES) pairing components of the GF

$$
\hat{G}_{\mathrm{ES}}^{\sigma}=\frac{1}{4} \sum_{i} \hat{\tau}_{i} \operatorname{Tr}\left[\check{\gamma}_{\sigma i} \check{G}_{\mathrm{loc}}\right] .
$$

Here, the projection operators to spin-up and spin-down states defined by the index $\sigma= \pm 1$ are given by $\check{\gamma}_{\sigma 0}=\hat{\tau}_{0} \hat{\sigma}_{0}+$ $\sigma \hat{\tau}_{3} \hat{\sigma}_{3}, \quad \check{\gamma}_{\sigma 1}=\hat{\tau}_{1} \hat{\sigma}_{1}-\sigma \hat{\tau}_{2} \hat{\sigma}_{2}, \quad \check{\gamma}_{\sigma 2}=\hat{\tau}_{2} \hat{\sigma}_{1}+\sigma \hat{\tau}_{1} \hat{\sigma}_{2}, \quad \check{\gamma}_{\sigma 3}=$ $\hat{\tau}_{3} \hat{\sigma}_{0}+\sigma \hat{\tau}_{0} \hat{\sigma}_{3}$. The generalized quasiclassical theory is formulated in terms of the spinless propagators

$$
\hat{g}_{\sigma}\left(\boldsymbol{n}_{p}, \boldsymbol{r}\right)=-\oint \frac{d \xi_{p \sigma}}{\pi i} \hat{G}_{\mathrm{ES}}^{\sigma}(\boldsymbol{p}, \boldsymbol{r}),
$$

where $\hat{G}_{\mathrm{ES}}^{\sigma}=\hat{G}_{\mathrm{ES}}^{\sigma}(\boldsymbol{p}, \boldsymbol{r})$ is the GF in the mixed representation, $\xi_{p \sigma}=p^{2} / 2 m_{F}+\sigma h-\mu$, and the notation $\oint$ means that the integration takes into account the poles of GF near the corresponding Fermi surface. Then, in the adiabatic approximation, which neglects the coupling between equal-spin and mixedspin correlations [41], we obtain the generalized KeldyshEilenberger equation:

$$
\begin{gathered}
i\left\{\hat{\tau}_{3} \partial_{t}, \breve{g}\right\}_{t}+i \boldsymbol{v}_{\sigma} \hat{\partial}_{\boldsymbol{r}} \hat{g}_{\sigma}-\left[\hat{\Sigma}_{\sigma}, \hat{g}_{\sigma}\right]_{t}=0, \\
\hat{\partial}_{\boldsymbol{r}}=\nabla-i e\left[\boldsymbol{A} \hat{\tau}_{3}, .\right]_{t}+i \sigma\left[\boldsymbol{Z} \hat{\tau}_{3}, .\right]_{t} .
\end{gathered}
$$

Here, the spin-dependent Fermi velocities $v_{ \pm}=$ $\sqrt{2(\mu \pm h) / m_{F}}$ are determined on each of the spin-split Fermi surfaces. The spin-dependent gauge field is given by the superposition of two terms $\boldsymbol{Z}=\boldsymbol{Z}^{m}+\boldsymbol{Z}^{\text {so }}$, where $Z_{i}^{m}=-i \operatorname{Tr}\left(\hat{\sigma}_{z} \hat{U}^{\dagger} \partial_{i} \hat{U}\right) / 2$ is the texture-induced part and the term $Z_{i}^{\text {so }}=m_{F}\left(\boldsymbol{m} \boldsymbol{B}_{i}\right)$ (where $\boldsymbol{m}=\boldsymbol{M} / \boldsymbol{M}$ ), which appears due to the SOC.

One can see that the Eilenberger-type equations for the spinup/down correlations contain an additional U(1) gauge field $\boldsymbol{Z}$ which is added to the usual electromagnetic vector potential $\boldsymbol{A}$ with the opposite effective charges for spin-up and spindown Cooper pairs. On a qualitative level, it is equivalent to the adiabatic approximation in the single-particle problems that allows to describe the quantum system evolution in terms of the Berry gauge fields [50].

\section{B. Charge and spin currents}

The Eilenberger equations (13) are supplemented by the expressions for the charge current $\boldsymbol{j}$ and the spin current $\boldsymbol{J}^{k}$, where $k$ denoted the spin index. The former is given by

$$
\boldsymbol{j}(t)=-\frac{\pi e}{4} \sum_{\sigma= \pm} v_{\sigma}\left\langle\boldsymbol{v}_{\sigma} \operatorname{Tr}\left[\hat{\tau}_{3} \hat{g}_{\sigma}^{K}(t, t)\right]\right\rangle,
$$

where $v_{\sigma}$ are the spin-resolved DOS and $\langle\cdots\rangle$ denotes the averaging over the spin-split Fermi surface.

The spin current in rotated frame is given by

$$
\tilde{\boldsymbol{J}}^{z}(t)=-\frac{\pi}{8} \sum_{\sigma= \pm} \sigma v_{\sigma}\left\langle\boldsymbol{v}_{\sigma} \operatorname{Tr}\left[\hat{\tau}_{3} \hat{g}_{\sigma}^{K}(t, t)\right]\right\rangle .
$$

\section{Diffusive limit}

Let us consider the system with large nonmagnetic impurity scattering rate as compared to the superconducting energies determined by the bulk energy gap $\Delta$. In this experimentally relevant diffusive limit, it is possible to derive the generalized Usadel theory with the help of the normalization condition $\left(\hat{g}_{\sigma} \circ \hat{g}_{\sigma}\right)\left(t_{1}, t_{2}\right)=\hat{\delta}\left(t_{1}-t_{2}\right)$, which holds due to the commutator structure of the quasiclassical equations (13).

The impurity self-energy in the Born approximation is given by $\hat{\Sigma}_{\sigma}=\left\langle\hat{g}_{\sigma}\right\rangle / 2 i \tau_{\sigma}$. In the dirty limit, we have

$$
2 \tau_{\sigma}\left(\boldsymbol{v}_{\sigma} \hat{\partial}_{\boldsymbol{r}}\right) \hat{g}_{\sigma}=-\left[\left\langle\hat{g}_{\sigma}\right\rangle, \hat{g}_{\sigma}\right]_{t} .
$$

The solution of Eq. (17) can be found as $\hat{g}_{\sigma}=\left\langle\hat{g}_{\sigma}\right\rangle+$ $\hat{\boldsymbol{g}}_{\sigma}^{a} \boldsymbol{p}_{\sigma} / p_{\sigma}$, where the anisotropic part of the solution $\hat{\boldsymbol{g}}_{\sigma}^{a}$ is small with respect to $\left\langle\hat{g}_{\sigma}\right\rangle$. Making use of the relation $\left\{\left\langle\hat{g}_{\sigma}\right\rangle, \hat{\boldsymbol{g}}_{\sigma}^{a}\right\}_{t}=$ 0 , which follows from the normalization condition, one obtains

$$
\hat{\boldsymbol{g}}_{\sigma}^{a}=-\tau_{\sigma} \boldsymbol{v}_{\sigma}\left\langle\hat{g}_{\sigma}\right\rangle \circ \hat{\partial}_{\boldsymbol{r}}\left\langle\hat{g}_{\sigma}\right\rangle .
$$

Substituting to Eq. (13) and omitting the angle brackets, we get the diffusion equation

$$
\left\{\hat{\tau}_{3} \partial_{t}, \hat{g}_{\sigma}\right\}_{t}-D_{\sigma} \hat{\partial}_{\boldsymbol{r}}\left(\hat{g}_{\sigma} \circ \hat{\partial}_{\boldsymbol{r}} \hat{g}_{\sigma}\right)=0,
$$

where $D_{\sigma}$ are the spin-dependent diffusion coefficients, in the isotropic case given by $D_{\sigma}=\tau_{\sigma} v_{\sigma}^{2} / 3$. This equation is a spinscalar equation, but cannot describe conventional spin-singlet superconducting correlations unlike the standard spin-scalar form of the nonstationary Usadel equation [51]. It is only applicable for strong ferromagnets and describes equal-spin triplet correlations residing at one and the same Fermi surface. Therefore this equation is a nonstationary generalization of the corresponding equations for homogeneous strong ferromagnets [52] and inhomogeneous strong ferromagnets [41].

The current and spin current are obtained by substituting expansion (18) to Eqs. (15) and (16):

$$
\begin{gathered}
\boldsymbol{j}=\frac{\pi e}{4} \sum_{\sigma= \pm} v_{\sigma} D_{\sigma} \operatorname{Tr}\left[\hat{\tau}_{3} \hat{g}_{\sigma} \circ \hat{\partial}_{\boldsymbol{r}} \hat{g}_{\sigma}\right], \\
\tilde{\boldsymbol{J}}^{z}=\frac{\pi}{8} \sum_{\sigma= \pm} \sigma v_{\sigma} D_{\sigma} \operatorname{Tr}\left[\hat{\tau}_{3} \hat{g}_{\sigma} \circ \hat{\partial}_{\boldsymbol{r}} \hat{g}_{\sigma}\right] .
\end{gathered}
$$

Further simplification can be obtained as follows. First, due to the normalization condition, we introduce the parametrization of Keldysh component in terms of the distribution function $\hat{g}_{\sigma}^{K}=\hat{g}_{\sigma}^{R} \circ \hat{f}_{\sigma}-\hat{f}_{\sigma} \circ \hat{g}_{\sigma}^{A}$. Then, switching to the mixed representation in time-energy domain $\hat{g}_{\sigma}\left(t_{1}, t_{2}\right)=$ 
$\int_{-\infty}^{\infty} \hat{g}_{\sigma}(\varepsilon, t) e^{-i \varepsilon\left(t_{1}-t_{2}\right)} d \varepsilon / 2 \pi$, where $t=\left(t_{1}+t_{2}\right) / 2$, we keep only the lowest order terms in the time derivatives.

As an example of the above procedure, one can obtain from (20) the charge current in the normal state $j=e^{2}\left(v_{+} D_{+}-\right.$ $\left.\nu_{-} D_{-}\right) \boldsymbol{E}_{e}$ driven by the emergent electric field $[19,53] \boldsymbol{E}_{e}=$ $-\partial_{t} \boldsymbol{Z}$. We, however, will neglect these effects and take into account only the quasiequilibrium contributions to the currents given by

$$
\begin{gathered}
\boldsymbol{j}=\sum_{\sigma= \pm} \frac{e v_{\sigma} D_{\sigma}}{8} \int_{-\infty}^{\infty} d \varepsilon f_{0} \operatorname{Tr}\left(\hat{\tau}_{3} \hat{\boldsymbol{J}}_{\sigma}^{\mathrm{RA}}\right) \\
\tilde{\boldsymbol{J}}^{z}=\sum_{\sigma= \pm} \frac{\sigma v_{\sigma} D_{\sigma}}{16} \int_{-\infty}^{\infty} d \varepsilon f_{0} \operatorname{Tr}\left(\hat{\tau}_{3} \hat{\boldsymbol{J}}_{\sigma}^{\mathrm{RA}}\right)
\end{gathered}
$$

where $\hat{\boldsymbol{J}}_{\sigma}^{\mathrm{RA}}=\hat{g}_{\sigma}^{R} \hat{\partial}_{\boldsymbol{r}} \hat{g}_{\sigma}^{R}-\hat{g}_{\sigma}^{A} \hat{\partial}_{\boldsymbol{r}} \hat{g}_{\sigma}^{A}$ is the spectral current and $f_{0}(\varepsilon)=\tanh (\varepsilon / 2 T)$ is equilibrium distribution function.

\section{Supercurrent-induced torque}

In the quasiequilibrium regime when the time derivative of the GF in the mixed representation can be neglected, Eqs. (13) or (19) yield the conservation of spin current in rotating frame $\nabla \cdot \tilde{J}_{z}=0$. The spin current in the laboratory frame is given by $\boldsymbol{J}^{k}=R_{k z} \tilde{\boldsymbol{J}}^{z}$, which can be written in the form

$$
\boldsymbol{J}^{k}(\boldsymbol{r})=m_{k}(\boldsymbol{r}) \tilde{\boldsymbol{J}}^{z} \text {. }
$$

It is not conserved due to the spatially-dependent magnetization of $d$ electrons $\boldsymbol{m}=\boldsymbol{m}(\boldsymbol{r})$.

Substituting Eq. (24) into Eq. (10), we obtain the torque, induced by the supercurrent in the quasiequilibrium regime:

$$
\begin{gathered}
J_{\text {sd }} \boldsymbol{M}_{s} \times \boldsymbol{M}=\boldsymbol{N}_{\mathrm{st}}+\boldsymbol{N}_{\mathrm{so}}, \\
\boldsymbol{N}_{\mathrm{st}}=2 \mu_{B}\left(\tilde{\boldsymbol{J}}^{z} \nabla\right) \boldsymbol{m}, \\
\boldsymbol{N}_{\text {so }}=4 \mu_{B} m_{F}\left(\boldsymbol{m} \times \boldsymbol{B}_{j}\right) \tilde{J}_{j}^{z} .
\end{gathered}
$$

Here, $\boldsymbol{N}_{\text {st }}$ is the supercurrent spin-transfer torque, which takes only the form of the adiabatic torque in the considered approximation, and $\boldsymbol{N}_{\text {so }}$ is the spin-orbit torque. Its particular structure strongly depends on the type of the spin-orbit coupling, realized in the system. Below we show that due to the coherent nature of the spin-polarized superconducting current the same result can be obtained from the energy functional of the system yielding the correction to the effective field.

\section{SUPERCURRENT SPIN TORQUES AS CORRECTIONS TO THE EFFECTIVE FIELD}

Above, we have derived general expressions (26) and (27) for the superconducting spin torques starting from the kinetic equation treated in the adiabatic limit. For any particular system, one can find the spin torques solving generalized Eilenberger/Usadel equations for the quasiclassical propagators and calculating the spin current according to Eq. (21).

An alternative approach to obtain superconducting spin torques is based on the description of magnetization dynamics in terms of the phenomenological expression for the effective field $\boldsymbol{H}_{\text {eff }}=-\delta F / \delta \boldsymbol{M}$, where $F=F(\boldsymbol{M})$ is the system energy as a functional of the magnetization distribution. The LLG equation without dissipation terms is given by

$$
\dot{\boldsymbol{M}}=-\gamma \boldsymbol{M} \times \boldsymbol{H}_{\mathrm{eff}} .
$$

This approach cannot be applied to derive spin-transfer torques in the normal state where the conduction electron magnetization is not coherent. In contrast to the normal system, superconducting electrons are in the macroscopically coherent state. Therefore the total energy of the system written in terms of the macroscopic variables describes the interaction between the condensate spin and the ferromagnetic order parameter.

Based on the above discussion one can conclude that the superconducting spin-transfer torques (26) and (27) can be obtained from the energy arguments. To demonstrate this, we consider a generic example of the Josephson system consisting of superconducting leads coupled through the ferromagnet with nonhomogeneous magnetization texture. In general, this task is rather complicated and requires extensive numerical calculations for each particular system considered. However, in strong ferromagnets, the general expressions for Josephson spin and charge currents for different magnetic textures of the interlayer can be obtained using the machinery of the generalized quasiclassical theory [41].

We consider the 1D magnetic texture $\boldsymbol{M}=\boldsymbol{M}(x)$ in the interlayer of the thickness $d$ between two superconducting interfaces, located at $x= \pm d / 2$. The superconducting order parameter phase difference between them is $\chi$. The currentphase relation for this setup has been found [41] as the superposition of partial currents carried by the spin-up and spin-down Cooper pairs:

$$
j(\chi)=\sum_{\sigma= \pm} j_{\sigma} \sin \left(\chi+2 \sigma \int_{-d / 2}^{d / 2} Z_{x} d x\right) .
$$

The amplitudes $j_{\sigma}$ are determined by the boundary conditions at FM/SC interfaces and the overlap factor of the equalspin correlations injected from the opposite SC electrodes $j_{\sigma} \propto e^{-d / \xi_{N \sigma}}$, where $\xi_{N \sigma}=\sqrt{D_{\sigma} / T}$ is the spin-dependent normal metal correlation length [41]. The other characteristic scale of the problem is the characteristic length of the magnetic inhomogeneity. In the case of the domain wall, it is the wall size $d_{w}$. If we are interested in the domain wall motion and consider the situation when the DW is located inside the interlayer $\left(d_{w}<d\right)$ and not in the vicinity of S/F interfaces, the amplitudes $j_{\sigma}$ do not depend on $d_{w}$ at all. However, this scale enters the Josephson current via the effective gauge field $\boldsymbol{Z}=$ $-m_{x}\left(m_{y} \nabla m_{z}-m_{z} \nabla m_{y}\right) / 2 m_{\perp}^{2}$, where $m_{\perp}=\sqrt{m_{y}^{2}+m_{z}^{2}} . Z$ is a crucial factor giving rise to the DW dynamics, as it is explained below. In more general case, when the DW is wide $d_{w}>d$ or the DW is located in the vicinity of a S/F interface, the amplitudes $j_{\sigma}$ also depend on $d_{w}$ via boundary conditions [41], but consideration of the DW dynamics presented below is not applicable in this case.

The rotated-frame spin current $\tilde{J}^{z} \equiv \tilde{J}_{x}^{z}$ is given by the difference of the partial spin-up/down currents

$$
\tilde{J}^{z}(\chi)=\frac{1}{2 e} \sum_{\sigma= \pm} \sigma j_{\sigma} \sin \left(\chi+2 \sigma \int_{-d / 2}^{d / 2} Z_{x} d x\right) .
$$


The Josephson energy can be obtained according to the usual relation

$$
F_{J}=\text { const }-\frac{1}{2 e} \sum_{\sigma= \pm} j_{\sigma} \cos \left(\chi+2 \sigma \int_{-d / 2}^{d / 2} Z_{x} d x\right)
$$

The current-phase relation (29) is given by $j(\chi)=$ $2 e\left(d F_{J} / d \chi\right)$. Therefore, calculating the correction to effective field $\tilde{\boldsymbol{H}}_{\text {eff }}=-\delta F_{J} / \delta \boldsymbol{M}$ (see details in Appendix), we obtain

$$
\gamma \boldsymbol{M} \times \tilde{\boldsymbol{H}}_{\mathrm{eff}}=2 \mu_{B} \tilde{\boldsymbol{J}}^{z}\left(2 m_{F} \boldsymbol{B}_{x} \times \boldsymbol{m}-\nabla_{x} \boldsymbol{m}\right) .
$$

Substituting the result (32) to the LLG equation, we get the spin-transfer torques identical to Eqs. (25)-(27).

The above energy consideration demonstrates that a direct coupling between the magnetization and superconducting current exist even in the limit when the spontaneous charge current is absent. Indeed, the spontaneous phase shift of the Josephson current-phase relation (29) is given by $\tan \chi_{0}=\tan \varphi\left(j_{+}-\right.$ $\left.j_{-}\right) /\left(j_{+}+j_{-}\right)$, where $\varphi=2 \int_{-d / 2}^{d / 2} Z_{x} d x$. Therefore $\chi_{0}=0$ in the limit when the spin subbands are formally degenerate, $j_{+}=j_{-}$. At the same time, the Josephson spin current (30) and correspondingly the spin torque in Eq. (32) are nonzero.

This result generalizes the previously suggested mechanism of the supercurrent-induced spin-orbital torque stemming from the $\chi_{0}=\chi_{0}(\boldsymbol{m})$ dependence $[31,32]$. In our case, it is not only the phase shift, but in addition the overall critical current in Eq. (29), which depends on the magnetization $j_{c}=$ $\sqrt{j_{+}^{2}+j_{-}^{2}+2 j_{+} j_{-} \cos (2 \varphi)}$ through $\varphi=\varphi(\boldsymbol{m})$. This provides the nonzero effective field even in the case of degenerate bands.

\section{SUPERCURRENT DRIVEN MAGNETIC TEXTURE DYNAMICS}

\section{A. General case of the texture dynamics driven by the adiabatic STT}

The first striking consequence of the dissipationless supercurrent spin torques is the possibility to realize the quasiequilibrium magnetic texture dynamics driven solely by the adiabatic STT generated by the superconducting current. In the absence of dissipation, the LLG equation (1) has a solution in the form of a traveling wave $\boldsymbol{m}=\boldsymbol{m}(x-u t)$ with a constant velocity determined by the spin current $u=2 \mu_{B} \tilde{J}^{z} / M$. For a periodic magnetic structure, e.g., magnetic helix, this yields a locally rotating magnetization with a frequency defined by $\omega \sim u / L$, where $L$ is the period. However, these timedependent quasiequilibrium solutions do not correspond to the ground state. It can be reached only in the presence of the Gilbert damping, which transforms the magnetic texture in such a way to compensate the effective field generated by the spin-polarized supercurrent. Therefore, eventually the systems will stop at the stationary state when $\boldsymbol{H}_{\text {eff }}=0$. In the absence of dissipation, the same quantity $u$ determines the characteristic velocity of the domain wall motion by the adiabatic STT in the system. In principle, current-driven motion of DWs in Josephson junctions with strong ferromagnets can be realized in different systems with high enough critical current densities. High critical currents through strong ferromagnets are typically carried by equal-spin triplet correlations, which decay on the length scale $\xi_{N \sigma}$ inside the ferromagnet [25]. The Josephson current carried through strong ferromagnets by equal-spin triplet pairs was experimentally reported in different systems [54-58] (see also Ref. [26] for review), which are the promising elements for the dissipationless superconducting spintronics. Here, we can estimate $u$ for the parameters of half-metallic $\mathrm{CrO}_{2}$ nanostructures [56]. The maximal Josephson current density through the $\mathrm{CrO}_{2}$ nanowire is $j_{c} \sim 10^{9} \mathrm{~A} / \mathrm{m}^{2}$, which determines the spin current $\tilde{J}^{z}=j_{c} /(2 e)$. Taking into account the saturation magnetization $M=4.75 \times 10^{5} \mathrm{~A} / \mathrm{m}$, we get the speed of the order of $u=1 \mathrm{~m} / \mathrm{s}$. As we show below, in case if the initial state contains DW, the ground state modified by the supercurrent can correspond either to the distorted DW or to the homogeneous state when the DW is eliminated from the sample. The dynamics of the initial state containing a DW under the applied supercurrent in the presence of the Gilbert damping in the LLG equation is also considered below.

\section{B. Domain wall motion}

Now we consider the magnetic texture of the ferromagnet in the form of the DW. We are interested in its dynamics induced by the supercurrent spin torques, discussed above. The two particular types of DW are considered: head-to-head DW and Neel DW.

The particular shape of the DW is dictated by the combination of the anisotropy energy and the exchange energy. We start with the head-to-head DW. In this case, the corresponding energy term can be written as follows:

$$
F=\frac{1}{2} \int d^{3} r\left[K_{\perp} m_{y}^{2}-K m_{x}^{2}+A_{\mathrm{ex}}\left(\nabla_{x} \boldsymbol{m}\right)^{2}\right],
$$

where $K>0$ and $K_{\perp}>0$ are the anisotropy constants for the easy and hard axes, respectively. $A_{\mathrm{ex}}$ is the constant describing the inhomogeneous part of the exchange energy. The effective magnetic field $\boldsymbol{H}_{\mathrm{eff}}=(1 / M)\left(K m_{x} \boldsymbol{x}-K_{\perp} m_{y} \boldsymbol{y}+A_{\mathrm{ex}} \nabla_{x}^{2} \boldsymbol{m}\right)$. It is convenient to parametrize the magnetization as follows:

$$
\boldsymbol{m}=(\cos \theta, \sin \theta \cos \delta, \sin \theta \sin \delta)
$$

where in general the both angles depend on $(x, t)$. At zero applied supercurrent, the equilibrium shape of the DW is given by $\delta=\pi / 2$ and

$$
\cos \theta= \pm \tanh \left[\left(x-x_{0}\right) / d_{w}\right]
$$

where $d_{w}=\sqrt{A_{\mathrm{ex}} / K}$ is the DW width. The above ansatz corresponds to the head-to-head DW, lying in the $x z$ plane. The tail-to-tail DW can be obtained by $\theta \rightarrow \theta+\pi$.

Let us consider the behavior of the head-to head DW under the applied supercurrent and the presence of SOC given by the superposition of the Rashba-type term $2 m_{F} \mu_{B} \boldsymbol{B}_{x} / M=$ $\left(0,-\beta_{R}, 0\right)$ and the Dresselhaus-type term $2 m_{F} \mu_{B} \boldsymbol{B}_{x} / M=$ $\left(\beta_{D}, 0,0\right)$.

First, we follow the Walker's procedure [59] by assuming that $\delta=\delta(t)$ and the DW is moving according to the timedependent shift $x_{0}(t)=\int_{0}^{t} v\left(t^{\prime}\right) d t^{\prime}$ in Eq. (35). Substituting this ansatz to the LLG equation, we obtain that this type of the solution exists only in the absence of Rashba SOC, $\beta_{R}=0$. We assume that the distortion of the wall is small during the wall motion, that is, $\delta=\pi / 2+\delta_{1}$, where $\left|\delta_{1}\right| \ll 1$. In this case, taking into account that $d_{w} \nabla_{x} \theta=\sin \theta$ for the DW, 
we obtain

$$
\begin{gathered}
\partial_{t} \delta_{1}=-\frac{\alpha v}{d_{w}}-2 \tilde{J}^{z} \beta_{D}, \\
\left(1+\alpha^{2}\right) v-u=\frac{\gamma d_{w} K_{\perp} \delta_{1}}{M}-2 d_{w} \tilde{J}^{z} \alpha \beta_{D} .
\end{gathered}
$$

In this case, Eqs. (36) and (37) yield the following equation for $v(t)$ :

$$
\partial_{t} v+\frac{\gamma \alpha K_{\perp}}{M\left(1+\alpha^{2}\right)} v=-\frac{2 d_{w} \gamma K_{\perp} u \beta_{D}}{M\left(1+\alpha^{2}\right)} .
$$

Taking into account the initial condition determined by Eq. (37), $\left(1+\alpha^{2}\right) v(t=0)=u-2 d_{w} \alpha \beta_{D} \tilde{J}^{z}$, which follows from $\delta_{1}(t=0)=0$, we determine the solution of Eq. (38) in the form

$$
\begin{gathered}
v(t)=\left[u+\frac{2 d_{w} \tilde{J}^{z} \beta_{D}}{\alpha}\right] \frac{e^{-t / t_{d}}}{\left(1+\alpha^{2}\right)}-\frac{2 d_{w} \tilde{J}^{z} \beta_{D}}{\alpha}, \\
\delta(t)=\frac{\pi}{2}+\frac{t_{d}\left(1-e^{-t / t_{d}}\right)}{1+\alpha^{2}}\left[\frac{u \alpha}{d_{w}}-2 \tilde{J}^{z} \beta_{D}\right] .
\end{gathered}
$$

where $t_{d}=\left(1+\alpha^{2}\right) M /\left(\alpha \gamma K_{\perp}\right)$ is the characteristic time scale. The solution for the moving DW expressed by Eqs. (39) and (40) exactly coincides with the solution found for the DW motion in normal ferromagnets under the influence of the adiabatic and nonadiabatic torques [60]. But, nevertheless, there is an important physical difference between the spin-orbit torque, considered here, and the nonadiabatic spin torque. As it can be seen from Eq. (27), the SO torque is equivalent to the torque, generated by an external applied field $\gamma \boldsymbol{H}=$ $-4 \mu_{B} m_{F} \tilde{J}_{j}^{z} \boldsymbol{B}_{j} / M$. Consequently, it moves DWs of opposite types $(+/-$ and $-/+)$ to opposite directions as opposed to the action of the nonadiabatic torque, which moves all the DWs in one and the same direction. At the same time, it is seen from Eqs. (36) and (37) that the Rashba SO torque is equivalent to the field perpendicular to the wall plane, therefore it does not move the DW and only distorts it. The solution (39) and (40) is only valid for small enough electric and, correspondingly, spin currents, applied to the system. If the current is large enough, the condition $\left|\delta_{1}\right| \ll 1$ is violated and Eqs. (36) and (37) are not valid. It was shown [15] that in this regime for $\tilde{J}^{z}>\tilde{J}_{\text {crit }}$ the DW can be moved even by the adiabatic torque only.

We consider the regime of arbitrary values of the applied current numerically by solving Eq. (2) together with the expressions for the torque, Eqs. (25)-(27), and the effective field $H_{\text {eff }}$, found from Eq. (33). The results for the case of small enough applied currents, when our analytical solutions are valid, are represented in Fig. 1. The figure demonstrates the displacement of the DW center as a function of time. The black curve corresponds to the case of no spin-orbit torque. The blue and pink curves are for the Rashba case, $\beta_{R} \neq 0, \beta_{D}=0$. They demonstrate that the Rashba spin-orbit torque does not move the DW in this case, as it was mentioned above. The green and red curves demonstrate the influence of the Dresselhaus SO torque on the DW motion. In agreement with our analytical calculations, the numerics gives that at $t \gg t_{d}$ the DW moves with a constant velocity. The direction of the motion is determined by the sign of $\beta_{D}$ or, in other words, by the sign of the effective magnetic field. In this case,

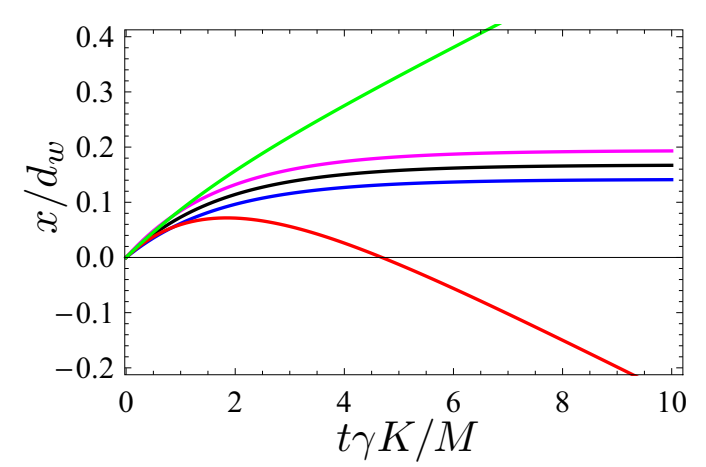

FIG. 1. The displacement of the DW as a function of time in the regime below the threshold current $\tilde{J}_{\text {crit }} . \beta_{R}=\beta_{D}=0$ (black), $\beta_{R}=$ $-0.05, \beta_{D}=0$ (blue), $\beta_{R}=0.05, \beta_{D}=0$ (pink), $\beta_{R}=0, \beta_{D}=$ -0.05 (green), $\beta_{R}=0, \beta_{D}=0.05$ (red). The other parameters are $K_{\perp} / K=3.0, \alpha=0.2$ and $\tilde{J}^{z}=-0.1 K d_{w}$ for all the curves.

it is possible that the DW reverses the direction of its motion if the adiabatic spin torque tends to displace it in the direction opposite to the one dictated by the effective field. This case is illustrated by the red curve in Fig. 1.

The regime of large applied currents $\tilde{J}^{z}>\tilde{J}_{\text {crit }}$, when the DW can be moved by the adiabatic torque only, is shown in Fig. 2. We have obtained that the value of $\tilde{J}_{\text {crit }}$ is rather close to $K d_{w}$. Therefore the critical electric current density is of the order $e K d_{w} / \hbar \sim 10^{10} \mathrm{~A} / \mathrm{m}^{2}$, which is an order of magnitude larger than the Josephson critical current obtained in experiment [56]. Again, the black curve in Fig. 2 shows the displacement of the DW in the absence of the SO torques. The initial dynamics of the DW at small $t$ coincides with Fig. 1, but at larger values of $t$ the situation changes, so that in this regime the DW moves, but its velocity is not constant. The Rashba SO torque does not cause any essential influence on the DW dynamics, as in the case of the small applied currents. However, the effect of Dresselhaus SOC is significant and at the first glance unexpected. Indeed, as shown in Fig. 1, the torque generated by this type of SOC, e.g., for $\beta_{D}<0$, moves the DW to the direction $x>0$. However, in the above-threshold regime, it can also reduce the averaged DW velocity (green

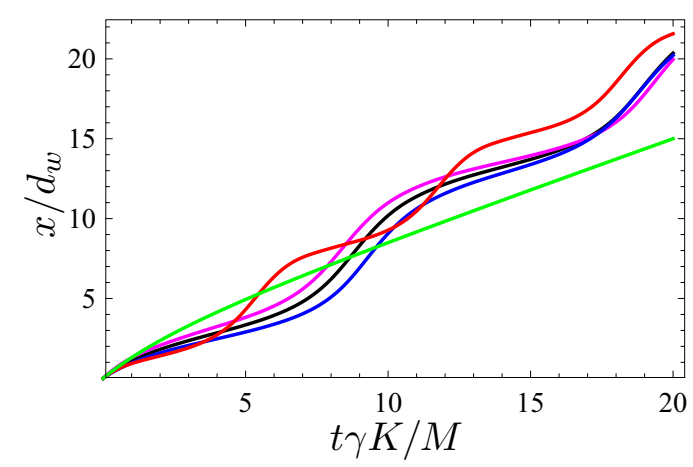

FIG. 2. The displacement of the DW as a function of time in the regime above the threshold current $\tilde{J}^{z}>\tilde{J}_{\text {crit }} . \beta_{R}=\beta_{D}=$ 0 (black), $\beta_{R}=-0.05, \beta_{D}=0$ (blue), $\beta_{R}=0.05, \beta_{D}=0$ (pink), $\beta_{R}=0, \beta_{D}=-0.05$ (green), $\beta_{R}=0, \beta_{D}=0.05$ (red). The other parameters are $K_{\perp} / K=3.0, \alpha=0.2$ and $\tilde{J}^{z}=-1.5 K d_{w}$ for all the curves. 

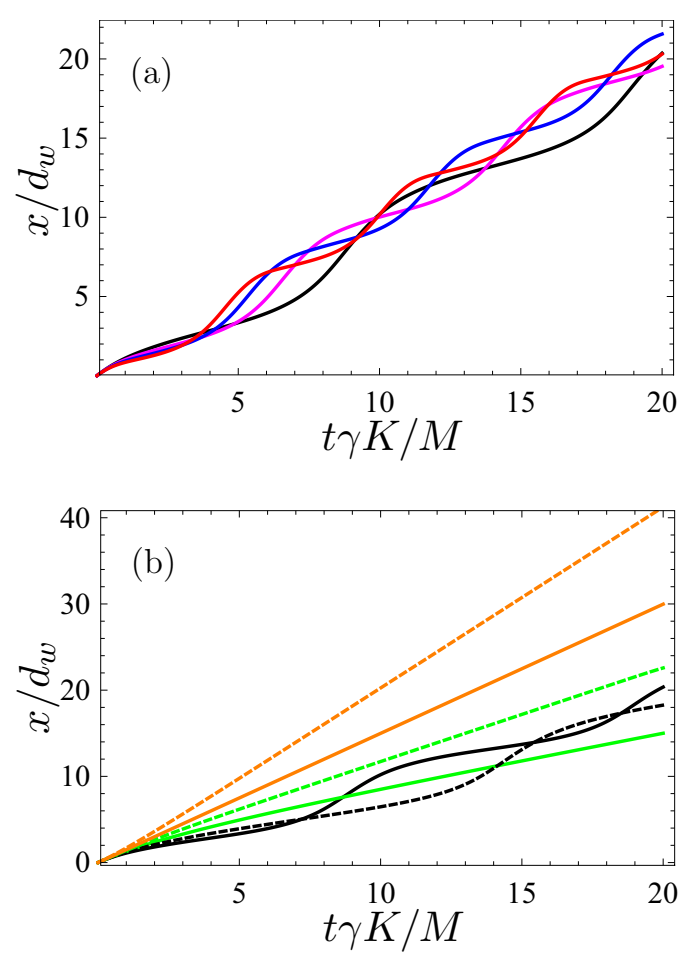

FIG. 3. The displacement of the DW as a function of time in the regime above the threshold current $\tilde{J}_{\text {crit }}$ for the case of $\beta_{R}=0$ and different values of the Dresselhaus SO coupling. (a) $\beta_{D}=0$ (black), 0.025 (pink), 0.05 (blue), 0.075 (red); (b) $\beta_{D}=0$ (black), -0.025 (dashed black), -0.05 (green), -0.075 (dashed green), -0.1 (tan), -0.15 (dashed tan). The other parameters are as in Fig. 2.

curve in Fig. 2), that is, the combined action of the adiabatic ST torque and SO torque cannot be viewed just as a simple sum of independent motions due to the both reasons. Vice versa, the SO torque generated at $\beta_{D}>0$, which by itself tends to move the DW to the direction $x<0$, can slightly enhance the average DW velocity, as it is demonstrated by the red curve.

The influence of the Dresselhaus SOC on the DW average velocity is represented in Fig. 3 in more detail. Figure 3(a) demonstrates the displacement of the DW as a function of time $t$ for several values of $\beta_{D}>0$. It is seen that there is a weak increase of the average velocity at $\beta_{D}>0$, but the more important and pronounced effect is that increasing $\beta_{D}$ leads to the decrease of the velocity oscillation period. The case $\beta_{D}<0$ is shown in Fig. 3(b), where one can see that the dependence of the average DW velocity on $\beta_{D}$ is nonmonotonous. While at smaller values of $\left|\beta_{D}\right|$ the average velocity is indeed reduced with respect to the case $\beta_{D}=0$, at larger values of $\left|\beta_{D}\right|$, the velocity starts to increase and exceed its value at $\beta_{D}=0$ considerably.

This behavior can be understood in the framework of the analogy between the SO torque and the magnetic-field induced torque. For the situation when the DW moves under the combined action of the current-induced torque and fieldinduced torque, it is known that the steady motion of the DW with $\dot{\delta}=0$ is only possible for a range of fields and currents [61]. The lines in the $\left(\tilde{J}^{z}, H\right)$ plane, separating the regions of steady motion and precession motion $\dot{\delta} \neq 0$, are called by the Walker-like stability lines [61]. This limit condition for the steady motion is strictly equivalent to the Walker breakdown [59] condition in the case where only an external magnetic field is applied. For the problem under consideration, the increase of $\beta_{D}$ absolute value at fixed current is equivalent to the increase of the applied field (at fixed current). When at zero $\beta_{D}$ the system is in the precession regime, as in Fig. 2, the increase of $\left|\beta_{D}\right|$ at $\beta_{D}<0$ moves the system towards the steady motion region, where the wall velocity is higher. Therefore the transition from the precession regime to the steady regime in Figs. 1 and 2 is analogous to crossing the Walker-like stability lines for problem of DWs motion under the combined action of the current-induced torque and field-induced torque.

Let us now consider the Neel DW. In this case, the combination of the anisotropy energy and the exchange energy takes the form

$$
F=\frac{1}{2} \int d^{3} r\left[K_{\perp} m_{z}^{2}-K m_{y}^{2}+A_{\mathrm{ex}}\left(\nabla_{x} \boldsymbol{m}\right)^{2}\right] .
$$

It is convenient to parametrize the magnetization as

$$
\boldsymbol{m}=(\sin \theta \sin \delta, \cos \theta, \sin \theta \cos \delta) .
$$

At zero applied supercurrent, the equilibrium magnetization profile is described by Eqs. (35) and $\delta=\pi / 2$. It can be shown that the problem of the Neel DW motion in the presence of the Rashba SO coupling is mathematically equivalent to the considered above motion of the head-to-head DW in the presence of the Dresselhaus SO coupling with the substitution $\beta_{D} \rightarrow-\beta_{R}$. Therefore, in this case, the Rashba SO torque plays the part of the field-induced torque moving DWs.

The above analysis demonstrates that the dynamics of a DW under an applied supercurrent depends strongly (i) on the particular type of the DW and (ii) on the particular type of the SO coupling, which induces the spin-orbit torque. The stationary motion of the DWs induced by small supercurrents is possible even in the absence of the nonadiabatic torque if the spin-orbit torque is present in the system.

Due to the presence of the Gilbert damping, the motion of a DW by a supercurrent is not a disspationless process. Interestingly, the DW motion generates voltage across the junction in the regime when the charge current is fixed but its magnitude is smaller than the Josephson critical current of the system. In this situation, the voltage can manifest itself as an additional step at the current-voltage characteristics of the junction at $j<j_{c}$, where $j_{c}$ is the critical current of the junction. The voltage amplitude $V$ can be roughly estimated from the balance of the energy dissipation rate in the magnetic subsystem due to the Gilbert damping and the power put in by the current source. The characteristic energy dissipation rate can be estimated as $\dot{F} \sim \Delta F / t_{d}$, where $\Delta F$ is the difference between the free energies of the equilibrium state of the DW at zero current and the nonequilibrium state of the distorted wall in the presence of the current. Our quasiequilibrium consideration of the DW dynamics is strictly valid only if $e V$ is small with respect to the characteristic inverse timescale of the problem $\gamma K / M$. For small distortions of the DW, $\Delta F$ can be obtained as follows:

$$
\Delta F=\frac{1}{2} \int d^{3} r\left[K_{\perp} \sin ^{2} \theta+A_{\mathrm{ex}}\left(\nabla_{x} \theta\right)^{2} \cos ^{2} \theta\right] \delta_{1}^{2} .
$$


Substituting the equilibrium profile of the DW $\theta(x)$ given by Eq. (35) into Eq. (43), we obtain

$$
\Delta F=S_{p} d_{w}\left(K_{\perp}+K / 3\right) \delta_{1}^{2},
$$

where $S_{p}$ is the cross-section area of the ferromagnet. The voltage, generated at the Josephson junction can be estimated as $V \sim \dot{F} / S_{p} j_{c}$, which yields

$$
V \sim \frac{\gamma \delta_{1}^{2} \alpha d_{w} K_{\perp}\left(K_{\perp}+K / 3\right)}{j_{c} M \hbar},
$$

where we have assumed that $\alpha \ll 1$.

For estimations, we use the material parameters of the $\mathrm{CrO}_{2}$ nanostructures [62], which are the promising systems for the dissipationless spintronics [56]. Taking the maximal Josephson current density through the $\mathrm{CrO}_{2}$ nanowire to be $j_{c} \sim 10^{9} \mathrm{~A} / \mathrm{m}^{2}$, the saturation magnetization $M=$ $4.75 \times 10^{5} \mathrm{~A} / \mathrm{m}, d_{w}=10^{-6} \mathrm{~cm}, K=1.43 \times 10^{5} \mathrm{erg} / \mathrm{cm}^{3}$, and $K_{\perp}=3 \mathrm{~K}$, we obtain $V \sim 0.1 \delta_{1}^{2} \mathrm{mV}$, where we took into account the typical values of the Gilbert damping $\alpha \sim$ 0.01 . The amplitude of DW distortion angle can be varied in wide limits, e.g., $\delta_{1}^{2} \sim 10^{-4}-10^{-3}$ for the red curve in Fig. 1, $\delta_{1}^{2} \sim 10^{-3}$ for the green curve in Fig. 1 and $\delta_{1}^{2} \sim 10^{-1}$ for the dashed green curve in Fig. 3(b). The estimated values of the induced voltage $V$ are small with respect to the characteristic superconducting scales $\sim 0.1 \mathrm{mV}$ for Al superconductors, therefore our assumption of quasiequilibrium quasiparticle distribution works rather well. From the other hand, the strict calculation of the voltage induced at the Josephson junction requires accounting for dynamics of the superconducting phase induced by the DW motion in the current-phase relation. This is beyond the scope of the present paper and will be done elsewhere.

\section{CONCLUSION}

To conclude, we have calculated the spin-transfer torques acting on the magnetic textures from the spin-polarized superconducting current flowing through the ferromagnetic material. For this, we take the advantage of the widely used adiabatic approximation, bringing it from the realm of single-electron dynamics into the field of superconductivity governed by the propagation of the spin-triplet Cooper pairs generated at the $\mathrm{SC} / \mathrm{FM}$ interface. This approximation enables us to find the analytical expression for the spin torques in the most general case of the spin texture and develop the efficient formalism of the generalized quasiclassical theory for calculating the charge and spin supercurrents through the inhomogeneous magnetic systems. We show that the supercurrent-driven dynamics of DWs crucially depends on the type and magnitude of the spin-orbital coupling. The obtained results demonstrate that the DW motion by the supercurrent is a phenomenon realistic for the recently developed Josephson junctions through $\mathrm{CrO} 2$ nanowires.

\section{ACKNOWLEDGMENTS}

This work was supported by the Academy of Finland Research Fellow (Project No. 297439) and RFBR Grant No.1802-00318. We thank Jan Aarts and Tero Heikkila for interesting discussions that initiated this project.

\section{APPENDIX: CALCULATION OF THE EFFECTIVE FIELD (32)}

From Eqs. (30) and (31), we obtain

$$
\frac{\partial F_{J}}{\partial \boldsymbol{M}}=\frac{2 \tilde{J}_{x}^{z}}{M} \frac{\delta \int_{-d / 2}^{d / 2} Z_{x} d x}{\delta \boldsymbol{m}} .
$$

Let us consider the following form of the unitary matrix $\hat{U}=\exp \left[-i \sigma_{x}\left(\frac{\delta}{2}+\frac{\pi}{4}\right)\right] \exp \left[-i \sigma_{y}\left(\frac{\theta}{2}+\frac{\pi}{4}\right)\right]$, which yields the texture part of the gauge field $\boldsymbol{Z}^{m}=-\cos \theta \nabla \delta / 2$, where $m_{x}=\cos \theta$ and $\tan \delta=m_{z} / m_{y}$ so that $\nabla \delta=\left(m_{y} \nabla m_{z}-\right.$ $\left.m_{z} \nabla m_{y}\right) / m_{\perp}^{2}$, where $m_{\perp}=\sqrt{m_{y}^{2}+m_{z}^{2}}$. Then we get

$$
\begin{gathered}
\frac{\delta}{\delta \boldsymbol{m}_{x}} \int_{-d / 2}^{d / 2} \boldsymbol{Z}^{m} d x=-\frac{\boldsymbol{x}}{2 m_{\perp}^{2}}\left(m_{y} \nabla m_{z}-m_{z} \nabla m_{y}\right), \\
\frac{\delta}{\delta \boldsymbol{m}_{y}} \int_{-d / 2}^{d / 2} \boldsymbol{Z}^{m} d x=-\frac{\boldsymbol{y}}{2 m_{\perp}^{2}} m_{z} \nabla m_{x}, \\
\frac{\delta}{\delta \boldsymbol{m}_{z}} \int_{-d / 2}^{d / 2} \boldsymbol{Z}^{m} d x=\frac{\boldsymbol{z}}{2 m_{\perp}^{2}} m_{y} \nabla m_{x} .
\end{gathered}
$$

Hence

$$
\begin{aligned}
& 2\left(\boldsymbol{M} \times \frac{\delta \int_{-d / 2}^{d / 2} Z_{x} d x}{M \delta \boldsymbol{m}}\right)_{y} \\
& \quad=\frac{1}{m_{\perp}^{2}}\left[m_{z}\left(m_{z} \nabla_{x} m_{y}-m_{y} \nabla_{x} m_{z}\right)-m_{y} m_{x} \nabla_{x} m_{x}\right]=\nabla_{x} m_{y} .
\end{aligned}
$$

Treating analogously other components and the spin-orbital part of the gauge field, we get

$$
\begin{gathered}
2\left(\boldsymbol{M} \times \frac{\delta \int_{-d / 2}^{d / 2} Z_{x}^{m} d x}{M \delta \boldsymbol{m}}\right)=\nabla_{x} \boldsymbol{m}, \\
2\left(\boldsymbol{M} \times \frac{\delta \int_{-d / 2}^{d / 2} Z_{x}^{\mathrm{so}} d x}{\boldsymbol{M} \delta \boldsymbol{m}}\right)=-2 m_{F} \boldsymbol{B}_{x} \times \boldsymbol{M} .
\end{gathered}
$$

Combining that into the total effective field yields Eq. (32).
[1] A. Brataas, A. D. Kent, and H. Ohno, Nat. Mater. 11, 372 (2012).

[2] N. Locatelli, V. Cros, and J. Grollier, Nat. Mater. 13, 11 (2013).

[3] E. Chen, D. Apalkov, Z. Diao, A. Driskill-Smith, D. Druist, D. Lottis, V. Nikitin, X. Tang, S. Watts, S. Wang, S. A. Wolf,
A. W. Ghosh, J. W. Lu, S. J. Poon, M. Stan, W. H. Butler, S. Gupta, C. K. A. Mewes, T. Mewes, and P. B. Visscher, IEEE Trans. Magn. 46, 1873 (2010).

[4] A. Yamaguchi, S. Nasu, H. Tanigawa, T. Ono, K. Miyake, K. Mibu, and T. Shinjo, Appl. Phys. Lett. 86, 012511 (2005). 
[5] J. A. Katine, F. J. Albert, R. A. Buhrman, E. B. Myers, and D. C. Ralph, Phys. Rev. Lett. 84, 3149 (2000).

[6] E. B. Myers, D. C. Ralph, J. A. Katine, R. N. Louie, and R. A. Buhrman, Science 285, 867 (1999).

[7] Y. Huai, F. Albert, P. Nguyen, M. Pakala, and T. Valet, Appl. Phys. Lett. 84, 3118 (2004).

[8] W.-G. Wang, M. Li, S. Hageman, and C. L. Chien, Nat. Mater. 11, 64 (2011).

[9] S. S. P. Parkin, M. Hayashi, and L. Thomas, Science 320, 190 (2008).

[10] M. Hayashi, L. Thomas, R. Moriya, C. Rettner, and S. S. P. Parkin, Science 320, 209 (2008).

[11] D. A. Allwood, Science 309, 1688 (2005).

[12] N. Vernier, D. A. Allwood, D. Atkinson, M. D. Cooke, and R. P. Cowburn, Europhys. Lett. 65, 526 (2004).

[13] A. Yamaguchi, T. Ono, S. Nasu, K. Miyake, K. Mibu, and T. Shinjo, Phys. Rev. Lett. 92, 077205 (2004).

[14] S. Zhang and Z. Li, Phys. Rev. Lett. 93, 127204 (2004).

[15] G. Tatara and H. Kohno, Phys. Rev. Lett. 92, 086601 (2004).

[16] A. Thiaville, Y. Nakatani, J. Miltat, and Y. Suzuki, Europhys. Lett. 69, 990 (2005).

[17] K. Obata and G. Tatara, Phys. Rev. B 77, 214429 (2008).

[18] I. M. Miron, T. Moore, H. Szambolics, L. D. Buda-Prejbeanu, S. Auffret, B. Rodmacq, S. Pizzini, J. Vogel, M. Bonfim, A. Schuhl, and G. Gaudin, Nat. Mater. 10, 419 (2011).

[19] N. Nagaosa and Y. Tokura, Nat Nano 8, 899 (2013).

[20] J. Iwasaki, M. Mochizuki, and N. Nagaosa, Nat. Commun. 4, 1463 (2013).

[21] A. Fert, V. Cros, and J. Sampaio, Nat. Nano 8, 152 (2013).

[22] F. S. Bergeret, A. F. Volkov, and K. B. Efetov, Phys. Rev. Lett. 86, 3140 (2001).

[23] F. S. Bergeret, A. F. Volkov, and K. B. Efetov, Phys. Rev. B 69, 174504 (2004).

[24] F. S. Bergeret, A. Levy Yeyati, and A. Martin-Rodero, Phys. Rev. B 72, 064524 (2005).

[25] F. S. Bergeret, A. F. Volkov, and K. B. Efetov, Rev. Mod. Phys. 77, 1321 (2005).

[26] M. Eschrig, Rep. Prog. Phys. 78, 104501 (2015).

[27] J. Linder and J. W. A. Robinson, Nat. Phys. 11, 307 (2015).

[28] J.-X. Zhu, Z. Nussinov, A. Shnirman, and A. V. Balatsky, Phys. Rev. Lett. 92, 107001 (2004).

[29] Z. Nussinov, A. Shnirman, D. P. Arovas, A. V. Balatsky, and J. X. Zhu, Phys. Rev. B 71, 214520 (2005).

[30] C. Holmqvist, S. Teber, and M. Fogelström, Phys. Rev. B 83, 104521 (2011).

[31] A. Buzdin, Phys. Rev. Lett. 101, 107005 (2008).

[32] F. Konschelle and A. Buzdin, Phys. Rev. Lett. 102, 017001 (2009).

[33] X. Waintal and P. W. Brouwer, Phys. Rev. B 65, 054407 (2002).
[34] J. Linder and T. Yokoyama, Phys. Rev. B 83, 012501 (2011).

[35] K. Halterman and M. Alidoust, Supercond. Sci. Technol. 29, 055007 (2016).

[36] I. Kulagina and J. Linder, Phys. Rev. B 90, 054504 (2014).

[37] J. Linder, A. Brataas, Z. Shomali, and M. Zareyan, Phys. Rev. Lett. 109, 237206 (2012).

[38] R. Takashima, S. Fujimoto, and T. Yokoyama, Phys. Rev. B 96, 121203 (2017).

[39] L. Berger, Phys. Rev. B 54, 9353 (1996).

[40] A. I. Buzdin, Rev. Mod. Phys. 77, 935 (2005).

[41] I. V. Bobkova, A. M. Bobkov, and M. A. Silaev, Phys. Rev. B 96, 094506 (2017).

[42] J. C. Slonczewski, J. Magn. Magn. Mater. 159, L1 (1996).

[43] T. Koyama, D. Chiba, K. Ueda, K. Kondou, H. Tanigawa, S. Fukami, T. Suzuki, N. Ohshima, N. Ishiwata, Y. Nakatani, K. Kobayashi, and T. Ono, Nat. Mater. 10, 194 (2011).

[44] I. M. Miron, Nat. Mater. 9, 230 (2010).

[45] P. Gambardella and I. M. Miron, Philos Transact A Math Phys Eng Sci 369, 3175 (2011).

[46] I. Garate, K. Gilmore, M. D. Stiles, and A. H. MacDonald, Phys. Rev. B 79, 104416 (2009).

[47] Edited by E. Y. Tsymbal and I. Zutic, Handbook of Spin Transport and Magnetism (CRC Press, Boca Raton, 2016).

[48] D. C. Ralph and M. D. Stiles, J. Magn. Magn. Mater. 320, 1190 (2008).

[49] A. Manchon and S. Zhang, Phys. Rev. B 78, 212405 (2008).

[50] K. Y. Bliokh and Y. P. Bliokh, Ann. Phys. 319, 13 (2005).

[51] A. I. Larkin and Y. N. Ovchinnikov, in Nonequilibrium Superconductivity, edited by D. N. Langenberg and A. I. Larkin (Elsevier, Dordrecgt, 1986).

[52] R. Grein, M. Eschrig, G. Metalidis, and G. Schon, Phys. Rev. Lett. 102, 227005 (2009).

[53] G. E. Volovik, J. Phys. C: Solid State Phys. 20, L83 (1987).

[54] T. S. Khaire, M. A. Khasawneh, W. P. Pratt, Jr., and N. O. Birge, Phys. Rev. Lett. 104, 137002 (2010).

[55] W. M. Martinez, W. P. Pratt, Jr., and N. O. Birge, Phys. Rev. Lett. 116, 077001 (2016).

[56] A. Singh, C. Jansen, K. Lahabi, and J. Aarts, Phys. Rev. X 6 , 041012 (2016).

[57] J. D. S. Witt, J. W. A. Robinson, and M. G. Blamire, Phys. Rev. B 85, 184526 (2012).

[58] J. W. A. Robinson, J. D. S. Witt, and M. G. Blamire, Science 329, 59 (2010).

[59] N. L. Schryer and L. R. Walker, J. Appl. Phys. 45, 5406 (1974).

[60] Z. Li, J. He, and S. Zhang, J. Appl. Phys. 99, 08Q702 (2006).

[61] A. Mougin, M. Cormier, J. P. Adam, P. J. Metaxas, and J. Ferré, Europhys. Lett. 78, 57007 (2007).

[62] X. Zou and G. Xiao, Appl. Phys. Lett. 91, 113512 (2007). 